\title{
Trivium
}

Revue franco-allemande de sciences humaines et sociales - Deutsch-französische Zeitschrift für Geistesund Sozialwissenschaften

$12 \mid 2012$

La sociologie de la culture en Allemagne

\section{Intérêts et idées. La problématique de l'imputation chez Max Weber}

\section{Rainer Lepsius}

Traducteur : Isabelle Kalinowski

\section{(2) OpenEdition}

Journals

Édition électronique

URL : http://journals.openedition.org/trivium/4368

DOI : $10.4000 /$ trivium.4368

ISSN : 1963-1820

Éditeur

Les éditions de la Maison des sciences de l'Homme

Référence électronique

M. Rainer Lepsius, «Intérêts et idées. La problématique de l'imputation chez Max Weber », Trivium [En ligne], 12 | 2012, mis en ligne le 20 décembre 2012, consulté le 07 septembre 2020. URL : http:// journals.openedition.org/trivium/4368; DOI : https://doi.org/10.4000/trivium.4368

Ce document a été généré automatiquement le 7 septembre 2020

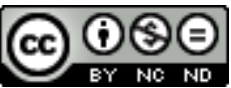

Les contenus des la revue Trivium sont mis à disposition selon les termes de la Licence Creative Commons Attribution - Pas d'Utilisation Commerciale - Pas de Modification 4.0 International. 


\title{
Intérêts et idées. La problématique de l'imputation chez Max Weber
}

\author{
M. Rainer Lepsius \\ Traduction : Isabelle Kalinowski
}

Nous remercions Monsieur M. Rainer Lepsius, la Kölner Zeitschrift für Soziologie und Sozialpsychologie ainsi que la maison d'édition Springer VS pour l'aimable autorisation de publier une traduction française de ce texte.

Wir danken Herrn M. Rainer Lepsius, der Kölner Zeitschrift für Soziologie und Sozialpsychologie sowie dem Verlag Springer VS für die freundliche Genehmigung, diesen Artikel in französischer Übersetzung zu publizieren.

1 Max Weber est considéré à juste titre comme le fondateur de la sociologie de la culture mais aussi comme son maître. Toute son œuvre, aussi bien la sociologie de la religion qu'Economie et société, est sous-tendue par l'effort d'analyser l'imbrication des intérêts et des idées. Sa sociologie fait constamment passer au premier plan deux problèmes : les intérêts, leur mise en forme, les conflits qui les opposent, et les idées, les conceptions de valeurs auxquelles se rapporte l'action des individus et des collectifs et qui peuvent servir à fonder et à légitimer les institutions. On cite toujours ce passage de Weber: «Ce sont les intérêts (matériels et idéels) et non les idées qui gouvernent directement l'action des hommes. Toutefois, les "images du monde" qui ont été créées par le moyen d' "idées" ont très souvent joué le rôle d'aiguilleurs, en déterminant les voies à l'intérieur desquelles la dynamique des intérêts a été le moteur de l'action ${ }^{1}$.» Dans ses analyses matérielles, Weber a concrètement mis en pratique la métaphore de "l'aiguillage ", et c'est là que réside aujourd'hui encore son actualité pour la sociologie de la culture. A partir de ses travaux - telle est l'hypothèse du présent article - on peut identifier un certain nombre de questions fondamentales pour les analyses de sociologie de la culture; ses méthodes peuvent suggérer des stratégies, permettre de cerner certaines difficultés et indiquer des pistes pour les résoudre.

2 La sociologie de la culture n'a pas pour mission de rappeler sans cesse que les idées, les conceptions de valeurs, la «culture » ont une importance pour l'action des hommes, pour l'organisation de leur vie en commun, pour leur style de conduite de vie, pour 
«l'habitus » de l'homme, pour "l'humanité » en général ou, selon les termes de René König, pour « l'auto-domestication de l'homme ». Ces énoncés découlent d'emblée des définitions anthropologiques qui identifient l'homme à un être "qui n'est pas limité à ses instincts ", qui est " ouvert au monde qui l'entoure » et capable de développer une structuration cognitive de sa réalité sociale. La tâche de la sociologie de la culture consiste plutôt à mettre en relation la logique de la structure de l'action et la logique de la construction du sens et, d'autre part, le comportement des hommes. Max Weber n'a cessé de thématiser ce problème de l'imputation. Dans la Remarque préliminaire aux Recueils d'études de sociologie de la religion, il se demande ainsi «par quel enchaînement de circonstances c'est sur le sol de l'Occident et nulle part ailleurs que sont apparus des phénomènes culturels qui s'inscrivaient pourtant - c'est du moins ainsi que nous aimons nous représenter les choses - dans une direction d'évolution dont la portée et la validité étaient universelles ${ }^{2} »$. Il a existé dans toutes les aires culturelles, écrit Weber, des rationalisations dans les différents domaines de la vie. "Pour caractériser ces différences dans la perspective de l'histoire culturelle, il faut se demander quelles sphères ont été rationalisées ${ }^{3}$. " Dans le conglomérat d'idées qui fait la culture d'une époque, il importe d'isoler le contenu de certaines d'entre elles et d'identifier leurs conséquences sociales. La sociologie est par suite confrontée à la nécessité de ne pas parler d'une culture en un sens vague et indéfini : elle doit prendre pour objet des idées précises. Elle doit développer la structure cognitive de ces dernières afin d'identifier, dans leurs traits caractéristiques, les composantes susceptibles d'infléchir les actions. C'est seulement lorsque ces conditions sont remplies que l'importance factuelle des idées pour l'action d'individus et de collectifs et pour l'institutionalisation de cette dernière peut être établie. Si les idées sont circonscrites de façon trop générale, si leur structure interne n'est pas définie, il est impossible de leur imputer des comportements sociaux. On en reste alors à l'approximation métaphorique d'une " affinité élective » ou à de vagues suppositions. La " culture » devient alors, en un sens, une «boîte noire", une catégorie résiduelle à laquelle on assigne tout ce qu'on n'a pu attribuer à d'autres facteurs. Plus les conceptions de valeur sont fluctuantes, plus leurs interprétations sont sujettes à variation, plus il est difficile de les définir comme des unités d'imputation potentielles. Par conséquent, les idées qui se prêtent le mieux à une définition exacte de la culture sont celles qui connaissent une concrétion dogmatique ou juridique, autrement dit les idées en usage dans les groupements religieux socialement établis ou dans les systèmes juridiques. Par suite, les terrains classiques de l'analyse des cultures sont la sociologie de la religion et la sociologie du droit.

3 Les écrits de sociologie religieuse de Weber sont une tentative de grande envergure pour mesurer la pertinence sociale des idées. Dans les "religions des cultures", on rencontre des idées suffisamment concrétisées d'un point de vue dogmatique, qui présentent, en outre, des variations notables si on les étudie dans une perspective de comparaison interculturelle. En même temps, Weber peut partir du principe que, «par le passé, les puissances magiques et religieuses et les représentations éthiques du devoir qui étaient ancrées dans la croyance en ces puissances contribuaient largement à modeler les conduites de vie ${ }^{4}$. Dans L'Ethique protestante et l'esprit du capitalisme, il tente d'aborder le problème «à partir de l'aspect le plus difficile à saisir: le rôle déterminant qu'ont joué certains contenus de croyance religieux dans la genèse d'une "mentalité économique", de "l' ethos" d'une forme économique ; l'exemple choisi ici sera celui du lien entre l'ethos économique moderne et l'éthique rationnelle du protestantisme ascétique ${ }^{5}$.» Weber cherche à identifier "l'enchaînement de 
circonstances » qui, d'un point de vue historique concret, a rendu possible, à partir des constructions religieuses du protestantisme ascétique, l'émergence d'une " conduite de vie pratique rationnelle» dont l'éthique professionnelle a marqué «l'ethos» d'une forme économique. A l'évidence, on a affaire ici à une longue chaîne de circonstances, à un processus complexe de médiation entre la structure d'une idée et les modes de comportement concrets portant la marque de cette dernière. Dans l'analyse de cette chaîne de circonstances, il importe d'établir « une imputation causale relativement univoque » du comportement économique concerné à la spécificité de ces idées. Telle est, de fait, la formulation la plus radicale du programme de la sociologie de la culture : étudier dans quelle mesure certains éléments du comportement social et de son organisation peuvent être rapportés à la spécificité de certaines idées. Weber lui-même a souligné à l'envi que ce programme ne présupposait pas une philosophie idéaliste de l'histoire.

4 Les idées peuvent naître dans un certain contexte et être l'expression de besoins psychiques et d'intérêts sociaux des types les plus divers. Une fois qu'elles existent, on peut se demander quel est l'effet qu'elles produisent sur les besoins psychiques et les formes de comportements sociaux. Les questionnements de la sociologie de la culture sont orientés dans deux directions : le contexte d'émergence des idées, d'une part, et, de l'autre, le contexte dans lequel elles exercent leurs effets. Les études de sociologie de la culture de Weber ont la particularité d'analyser concrètement les processus de "concaténation ». Tout à l'opposé des raccourcis d'une critique de la culture ou de l'ambition globale d'une philosophie de la culture, il s'agit pour lui d'étudier le rapport d'imputation empiriquement constatable qui rattache des phénomènes sociaux à des composantes culturelles. En ce sens, l'étude de Weber sur l'éthique protestante est un classique. Elle en est un pour des raisons méthologiques et heuristiques, indépendamment de la pertinence, ou non, des configurations historiques qu'il identifie et des imputations causales dont il formule l'hypothèse.

C'est sous cet aspect que nous examinerons une nouvelle fois les études de Weber sur l'éthique protestante, afin d'en tirer des indications systématiques pour un programme de sociologie de la culture. Weber lui-même affirmait que son «étude pourrait contribuer [...] à montrer comment les "idées" agissent dans l'histoire ${ }^{6} »$ : or, c'est précisément là la question centrale de la sociologie de la culture. Le point de départ de toute recherche de ce type est la détermination précise de l'idée dont on se propose d'analyser les effets sociaux. Cette définition précise fait trop souvent défaut, et il manque alors d'emblée une donnée de référence bien définie à laquelle imputer les effets observés. Pour prendre un exemple tiré d'un débat actuel, la notion de culture politique est le plus souvent utilisée dans un sens mal défini et elle englobe trop d'éléments contradictoires entre eux : par suite, les tentatives pour rapporter certains types de comportements à une culture politique ne donnent que des résultats insatisfaisants. Weber était conscient de cette difficulté. Après les passages introductifs sur les liens entre protestantisme et développement de l'économie capitaliste, il écrit : "Toute une série de connexions possibles se profilent obscurément sous nos yeux dès lors que nous posons la question en ces termes. Notre tâche devra précisément consister à formuler ce dont nous avons confusément l'intuition aussi clairement que l'inépuisable multiplicité inhérente à tout phénomène historique le permet. Cela suppose nécessairement d'abandonner le terrain des représentations vagues et générales dans lequel nous nous sommes jusqu'ici cantonné ; il faut à présent tenter de pénétrer la spécificité et les traits distinctifs des grands univers de pensée religieux qui 
se présentent à nous, historiquement, dans les différents courants de la religion chrétienne ${ }^{7}$.»

6 La tâche qui en résulte pour le sociologue de la culture consiste à mettre au jour ces idées et leur contenu, les conjonctions dans lesquelles elles orientent les comportements et, enfin, leur structure cognitive. Ceci outrepasse généralement la compétence spécialisée du sociologue, qui doit faire appel à des experts des domaines culturels concernés; cependant, il doit être en mesure d'élaborer ces résultats pour qu'ils deviennent exploitables dans l'optique des questionnements qui sont les siens. Cela signifie, au demeurant, qu'il doit acquérir lui-même un degré élevé de savoir sur ces domaines culturels. Weber offre un exemple remarquable des efforts qui peuvent être accomplis en ce sens : il avait acquis un savoir impressionnant dans le domaine de la science comparée des religions. L'état actuel de la sociologie de la culture, si souvent déploré, tient peut-être justement en partie au fait que les efforts auxquels il faut consentir pour acquérir des connaissances matérielles dans les domaines culturels concernés sont trop importants ou perçus comme tels.

7 L'idée choisie par Weber et dont il veut étudier les effets est « l'éthique professionnelle du protestantisme ascétique ». Cette éthique du métier est définie plus précisément comme un devoir de confirmation de l'individu dans le travail quotidien, quel qu'en soit le contenu et quelle que soit sa visée. Elle est associée à une conduite de vie ascétique globale conjuguant épargne, conscience professionnelle et auto-discipline. Au premier abord, il est tout à fait indifférent de savoir comment cette idée est née ; il suffit qu'elle se soit constituée indépendamment des variables sur lesquelles elle est censée exercer une influence. La singularité de cette éthique professionnelle spécifique ne découle pas de ses conséquences, par exemple de celles qu'elle a entraînées pour le développement du régime économique capitaliste, mais d'une construction de sens interne. La conduite de vie ascétique et sa visée de confirmation intramondaine au quotidien trouvent leur justification en tant que « cause de connaissance » de l'état de grâce personnel, de la "vocation personnelle à la félicité ${ }^{8}$ ». L'objectif de l'éthique du travail n'est pas une finalité extérieure, mais la certitude intérieure quant à l'état de grâce dans le cadre de la doctrine de la prédestination, qui n'autorise en aucun cas à tenter d'infléchir le plan de salut décidé par Dieu en ayant recours aux bonnes œuvres ou à des pratiques magiques. L'éthique professionnelle du protestantisme ascétique exige comme un devoir constant et systématique l'exercice d'un métier, qui constitue une valeur en soi et revêt une signification extraordinairement décisive pour l'action, dans la mesure où la connaissance de l'état de grâce personnel intéresse l'individu au plus haut point et où il croit aux attentes transcendantes de la grâce divine.

8 L'idée ainsi définie possède une série de propriétés qui sont de la plus haute importance pour les recherches en sociologie de la culture. Elle appelle un comportement spécifique, elle peut être distinguée d'autres éthiques du métier et mise en relation avec des groupes bien délimités. Il est nécessaire d'établir ces caractéristiques pour isoler, sur le plan cognitif dans un premier temps, l'idée dont les conséquences doivent être étudiées. L'idée qui peut être isolée sur le plan cognitif doit également avoir des destinataires spécifiques, chez lesquels on peut mesurer l'impact de sa revendication de validité. Le lien entre l'idée et le groupe de porteurs qui orientent leur comportement en fonction de celle-ci doit être suffisamment fort. Dans le cas de Weber, que nous avons pris ici pour exemple, ce lien résulte, en premier lieu, de l'ancrage existentiel de l'idée, qui est internalisée et sanctionnée par les croyants eux-mêmes. Ici, l'une et 
l'autre, la socialisation et la sanction de l'idée, sont l'œuvre du même groupe de porteurs : les croyants. De là découle la possibilité d'isoler, d'un point de vue social, la revendication de validité de l'idée. Le protestantisme ascétique a entraîné, il est vrai, une individualisation impitoyable et une destruction radicale des hiérocraties, mais il a préservé la religiosité communautaire. Le principe de cette dernière et la formation de sectes comme libre rassemblement de personnes égales en qualification du point de vue éthique permettent de rapporter, dans ce cas, des idées spécifiques à des groupes de porteurs spécifiques. Ce sont les membres des sectes eux-mêmes qui se socialisent et se sanctionnent mutuellement, ce qui garantit aussi bien l'identité que le caractère contraignant de la norme de comportement de ces groupes de porteurs. La force de la socialisation et la pression de la sanction assurent, dans le cas des sectes protestantes, un rapport immédiat entre la revendication de validité d'une conception de valeur spécifique et le comportement d'un groupe de porteurs donné. L'efficacité sociale de l'idée peut ainsi être observée dans les comportements factuels des membres de la secte. Dans les cas où une idée a été isolée d'un point de vue cognitif mais où son domaine de validité n'a pu être isolé d'un point de vue social, l'imputation des comportements observés aux idées en question soulève de grandes difficultés, car la question de savoir chez qui les idées ont acquis une force contraignante suffisante pour modeler les comportements n'est aucunement résolue. Les sectes et groupes du même type, dont le rapport spécifique aux valeurs est bien établi, sont de ce fait des objets particulièrement appropriés pour une étude de l'efficacité des idées. Toute la vie de leurs membres est infléchie par une éthique spécifique ; la visibilité du comportement de chaque individu dans de petits groupes permet un travail d'éducation constant et un contrôle immédiat. A cela s'ajoute, dans le cas des sectes protestantes, la possibilité d'une sanction grave, la privation de la communion, qui implique l'exclusion de la communauté des fidèles. Weber l'évoque dans l'« Anticritique finale à propos de L'Esprit du capitalisme $»^{9}$.

9 Les conséquences de l'éthique ascétique du métier résident tout d'abord en elle-même. Elle soumet à une perception planifiée et autoréflexive un certain champ de comportements -à savoir l'activité professionnelle - qui était jusque là situé à l'écart des normes ascétiques, voire carrément honni à bien des égards. Le tournant qui fait passer de l'ascèse extramondaine à l'ascèse intramondaine constitue le noyau théologique de la nouvelle éthique professionnelle ${ }^{10}$. Il en résulte la soumission du travail à une discipline considérablement plus stricte, son intensification et, conjointement, l'oblitération d'autres domaines de la vie. L'éthique professionnelle spécifique du protestantisme ascétique opère une sélection, détermine la direction de la conduite de vie, entraîne, positivement, la sélection de certains domaines de vie et, négativement, l'exclusion de certains autres, ainsi que des types de disciplines qu'ils exigent. Cette détermination de la direction est le fait de la structure cognitive de l'idée et elle est indépendante des conditions de vie des personnes concernées. L'idée peut s'accorder avec les intérêts, ils peuvent s'adapter les uns aux autres au fil du temps, mais ce qui est décisif, c'est que le comportement postulé dérive de la construction de l'idée elle-même. Le fait que ce postulat de comportement parvienne ou non à s'imposer peut dépendre de la possibilité de l'adopter en pratique dans la vie, mais il se fonde au premier chef sur la logique de l'idée. L'éthique ascétique du métier est dissociée de façon particulièrement nette des intérêts traditionnels et universels ; par rapport à ces derniers, elle apparaît comme irrationnelle, souligne Weber, et comme « la forme la plus intolérable de contrôle de l'Eglise sur l'individu ${ }^{11}$ ». Compte tenu de la 
plasticité de l'action humaine, la marge de variation des alternatives d'action qui peuvent être sélectionnées par les idées est manifestement importante.

Quant aux conséquences qui en résultent, elles ne dépendent pas seulement, il est vrai, de la construction cognitive de l'idée. Les propriétés du contexte au sein duquel s'inscrit le comportement sélectionné par l'idée jouent également un rôle déterminant. Si l'éthique ascétique du métier touche un groupe de porteurs dont l'activité s'exerce dans un domaine économique où les rapports d'échange sont organisés sur la base d'une économie monétaire, le travail professionnel constant et planifié peut déboucher sur des chances de profits. Les gains économiques ne sont pas l'objectif de l'idée, mais dans de telles conditions et dans un tel contexte, ils en sont la conséquence. Cette chance de faire des profits, d'accéder à l'aisance matérielle, est confortée par le fait que l'interdit de consommation de l'éthique ascétique du métier impose le réinvestissement des gains, à l'exclusion de tout autre usage ${ }^{12}$. Une conséquence secondaire de l'idée est l'accroissement des chances d'auto-financement d'entreprises nouvelles et plus étendues. La conséquence de l'éthique professionnelle est donc, directement, une augmentation de l'intensité du travail et, indirectement, une augmentation des chances de profit; directement, un renoncement à la consommation des gains obtenus et, indirectement, une augmentation du capital constitué et de l'auto-financement. Il faut donc distinguer des conséquences directes et indirectes; les conséquences directes peuvent être rapportées de façon immédiate à la pertinence sociale de l'idée, les conséquences indirectes sont quant à elles déterminées par les propriétés du contexte dans lequel l'idée agit. Dans ce contexte, l'idée ouvre la voie à d'autres relations fonctionnelles, par exemple celle qui associe l'épargne et l'investissement, c'est-à-dire l'augmentation du taux d'investissement, ou encore le financement propre et le financement extérieur, c'est-à-dire l'augmentation du taux de capital propre. A quoi s'ajoute, dans le cas des puritains, le gain de crédit personnel apporté par l'appartenance à une secte. A une époque où le crédit personnel est prédominant, la décision d'accorder un crédit dépend principalement des propriétés de la personne de l'emprunteur; or, l'appartenance à une secte prônant une éthique ascétique du métier garantit la conduite de vie et certifie que la personne concernée est digne de crédit ${ }^{13}$. Aux chances d'accroître l'auto-financement s'ajoutent donc celles d'améliorer les possibilités de financement extérieur. Là encore, il ne s'agit pas d'une conséquence directe de la structure cognitive de l'idée, mais d'un effet des configurations fonctionnelles de la société qui existent indépendamment de cette idée et de la pertinence de cette dernière pour les comportements.

11 Pour la sociologie de la culture, le problème est dès lors avant tout, à l'évidence, d'imputer de façon pertinente ces différents critères contingents. Les idées ont des conséquences qui dérivent de leur structure et des conséquences qui résultent seulement de la rencontre avec un contexte et des conditions spécifiques. Les différentes relations fonctionnelles doivent être soigneusement distinguées et spécifiées. Au demeurant, il ne faut pas seulement tenir compte des conséquences "non visées", dans une perspective intentionnelle, mais aussi, dans une perspective fonctionnelle, du déclenchement ou de l'inhibition de conséquences "secondaires ». Weber ne cesse de souligner que l'éthique protestante n'a pas eu le capitalisme pour visée, n'a pas déclenché à elle seule son émergence, qu'elle n'est qu'un des nombreux éléments qui ont favorisé son développement, mais qu'elle a cependant été d'une importance décisive pour la constitution du capitalisme rationnel d'entreprise. «Ce qui s'est opéré précisément, c'est la conjonction entre un écheveau de contenus psychiques 
issus de racines éthico-religieuses très spécifiques et des possibilités de développement capitaliste ${ }^{14}$.» Il s'agit manifestement d'analyser ces «conjonctions» de façon différenciée et de mettre au jour leur structure. La problématique de l'imputation se complique ainsi considérablement. D'un côté, les propriétés du contexte d'action rendent possible l'application d'impératifs de comportement qui découlent des idées; de l'autre, les nouveaux modes de comportement déclenchent l'activation de liens fonctionnels contenus dans le contexte d'action mais sans rapport aucun avec les idées. Plus les chaînes d'inductions mutuelles s'allongent, plus il devient difficile de distinguer les cas où des idées ont été rendues possibles par des conditions et un contexte et, d'autre part, ceux où les conditions et le contexte ont été transformés par des idées.

12 Le pouvoir des idées est particulièrement manifeste dans le cas des innovations qui peuvent être observées dans le temps et les structures. Toute innovation est d'abord un comportement déviant qui se trouve en contradiction avec les normes traditionnelles et subit d'emblée, par suite, la pression d'une sanction. L'importance de la conception de valeur qui déclenche l'innovation tient à sa double fonction de légitimation du comportement déviant. En premier lieu, celui qui innove et commet un écart se sent personnellement légitimé dans son action en se référant à ce fondement de valeur; en second lieu, il peut, dans certains cas, en invoquant ces conceptions de valeur, écarter, éluder et délégitimer la sanction que son environnement menace de prendre à l'encontre de son action. Pour l'imposition d'une innovation, l'assurance personnelle du novateur est d'une grande importance. C'est justement dans cette perspective que Weber souligne les effets de l'éthique protestante du métier. "L'entrepreneur [était empli] d'une bonne conscience inentamée, il était persuadé que, si la Providence lui avait montré la voie du profit, ce n'était pas sans une intention particulière: elle voulait qu'il la suive pour la gloire de Dieu ${ }^{15}$. » Il ne connaît pas le doute quant à la justification de son action et quant à la légitimité des résultats que celle-ci entrâne pour lui. « La profession et le noyau éthique le plus intime de la personnalité - c'est là le point décisif - forment ici une unité indissociable ${ }^{16}$. "Cette "bonne conscience» confère aussi à l'innovation, sur le plan de la personnalité, une force d'imposition extraordinaire et une capacité de résistance, y compris contre les jugements négatifs venus de l'extérieur et contre les menaces de sanctions. Le mode de légitimation par la valeur est en ce sens - tout à fait indépendamment du contenu de l'innovation de comportement - un moyen essentiel d'imposition d'une nouveauté, de dépassement des habitudes traditionnelles et de renversement des barrières représentées par les sanctions. La "tension entre la "conscience" et "l'agir" 17 " ne peut être surmontée que grâce à des conceptions de valeur spécifiques; c'est là que réside l'importance spécifique des idées, y compris pour l'imposition d'innovations. Cette importance s'accroît lorsque l'environnement concerné par ces innovations, dans ses intérêts et habitudes, reconnaît lui-même la conception de valeur au moyen de laquelle l'innovation cherche à se légitimer. Même si ses intérêts sont fortement concernés, il ne peut contester le fondement de valeur et la justification que celui-ci apporte à l'innovation. La croyance dans les idées transcende l'antagonisme des intérêts. Dans le cas des puritains, on trouve, à côté de l'entrepreneur qui se justifie lui-même, le travailleur à domicile ou l'ouvrier qui partage la même éthique professionnelle et exerce son métier avec une discipline de travail et de façon consciencieuse, sans remettre en cause la légitimé de l'entrepreneur ${ }^{18}$. Dans ce cas, légitimité interne et légitimité externe concordent directement. Mais même dans les contextes où l'éthique 
professionnelle n'était pas partagée, son fondement transcendant lui conférait de très fortes chances de s'imposer, en un temps où les fondements transcendants étaient ceux qui possédaient la crédibilité la plus forte. Même si l'innovation dissout les conceptions de valeur traditionnelles, la rupture avec la tradition ne réussit jamais aussi bien que lorsqu'elle s'opère au nom de conceptions de valeur traditionnellement admises. De ce fait, l'efficacité des idées n'est jamais aussi manifeste que dans des situations d'innovation, lorsqu'elles apportent un soutien et une motivation au novateur, et une caution et une légitimation vis-à-vis de l'extérieur. Les changements de comportement initiés par des minorités peuvent demeurer à l'état latent s'ils ne sont pas réprimés dès la phase de leur genèse et résorbés dans la succession des générations. Ils mènent alors, de leur côté, une existence marginale et tolérée. Beaucoup de sectes religieuses ont suivi cette voie, se sont perpétuées pendant des générations sans provoquer un changement général des orientations de comportement. Elles demeurent centrées sur leurs idées religieuses constitutives, se retirent du monde ou s'immunisent contre lui, se protégeant ainsi des sanctions extérieures et perpétuant la consonance cognitive dans leur milieu intermédiaire. Dans le cas des sectes protestantes ascétiques, cependant, Weber affirme qu'une nouvelle "mentalité économique " s'est généralisée et s'est diffusée de manière décisive, en l'occurrence, justement, "l'esprit du capitalisme ». On aborde ici une problématique centrale pour toute sociologie de la culture: la question de la diffusion des idées. Au premier abord, la diffusion des orientations de valeur semble pouvoir s'expliquer simplement par la conversion d'un nombre croissant de personnes aux idées de valeur des groupes qui en sont originellement les porteurs. Pourtant, dans le cas de l'éthique protestante du métier, ce n'est justement pas le cas. La mentalité économique capitaliste se diffuse sans que les sectes protestantes connaissent une augmentation correspondante du nombre de leurs membres. L'éthique professionnelle pratiquée dans un premier temps par une minorité en référence explicite à une valeur doit donc connaître une chance de diffusion qui ne soit pas tributaire de la socialisation des idées de valeur religieuses constitutives de cette éthique professionnelle. Nous touchons ici au problème des conséquences d'une idée qui, dans le même temps, perd sa validité. Weber évoque avec insistance cette conjonction. On ne saurait affirmer, écrit-il, "que l'appropriation subjective de ces maximes éthiques [celles de l'éthique protestante du métier, N.d.A] par ceux qui en sont les porteurs individuels, les entrepreneurs ou les ouvriers des entreprises capitalistes modernes, par exemple, soit à l'heure actuelle la condition de la survie du capitalisme ${ }^{19}$ ». «Voilà bien longtemps que le capitalisme moderne [...] n'a plus besoin de ce soutien ${ }^{20}$. » Des conceptions de valeur spécifiques possèdent, dans le contexte d'émergence de nouvelles formes de comportement, une autre fonction que celle qui leur revient lorsque ces formes de comportement ont été institutionnalisées.

Dans le sillage de l'institutionnalisation du comportement économique capitaliste, les justifications de valeur religieuses d'abord invoquées pour ce dernier font place à d'autres normes. L'autodiscipline et le contrôle de l'action, si caractéristiques de l'éthique protestante du métier, adoptent des critères qui sont assortis des mêmes effets, sans entretenir le moindre lien avec la signification que possédait, à l'origine, cette discipline personnelle. Ce qui peut être considéré comme un changement de valeurs, voire comme une décadence de la conviction morale n'est que le résultat de la routinisation de ce qui était, à l'origine, une morale de virtuoses. Celle-ci n'est plus nécessaire lorsqu'à la phase de genèse d'un nouveau mode de comportement succède son institutionnalisation. Le déclin de la puissance de validation associée à la 
justification première par une certaine valeur n'est pas, dans ce cas, l'expression d'une décadence culturelle, mais d'une diffusion réussie. Toute diffusion d'une morale de virtuoses, autrement dit toute extension de sa validité, va de pair avec une routinisation, c'est-à-dire un affaiblissement moral ; il en va de même de l'éthique du métier propre aux virtuoses de la morale des sectes protestantes.

La diffusion réussie d'un mode de comportement sans que soit perpétuée la valeur originellement invoquée pour le justifier est une question importante pour la problématique de l'imputation des effets et des conséquences des idées. Ce transfert est grandement facilité quand les résultats des nouveaux modes de comportement peuvent servir des objectifs qui n'étaient pas visés par l'idée d'origine. Alors que l'éthique protestante du métier considérait l'activité en elle-même comme une valeur, tout à fait indépendamment de ses résultats, le même type de comportement au travail fut ensuite apprécié en raison de ses succès. Les fondements de valeur d'un même comportement peuvent changer radicalement sans que ces changements trouvent une expression dans ce dernier. Ainsi, dans le processus de diffusion, l'éthique du métier est modifiée, mais la discipline du comportement de travail est préservée. Le fondement de valeur qui voit dans l'activité professionnelle disciplinée et auto-réflexive une base de connaissance de l'état de grâce peut être remplacé par l'idée sécularisée de l'autoréalisation de l'homme au moyen du produit de son travail, sans que le caractère du devoir de travail ne soit modifié en quelque façon. Pour finir, cependant, de simples critères d'efficacité et de succès peuvent suffire à légitimer l'éthique de travail, voire l'habitude que l'on a prise de modèles organisés d'exercice du travail. Dans tous ces cas, on a affaire à un changement dans les conceptions de valeur mais celles-ci ne pourraient être déduites d'un examen extérieur du comportement en question. Le passage qui s'opère entre un comportement infra-institutionnel en usage dans de petites communautés de socialisation et de sanction fortement structurées, telles que les sectes, et le comportement institutionnalisé de vastes groupes de population dans des contextes fonctionnels différenciés, comme c'est le cas dans le système économique capitaliste, recouvre des processus sociaux multiples et complexes. Ce qui nous intéresse avant tout ici, c'est la question de l'importance des idées dans ces derniers. Les conceptions de Weber relatives à l'importance de l'éthique protestante du métier pour la mentalité économique capitaliste se fondent sur l'hypothèse que le protestantisme ascétique a entraîné la rationalisation de "sphères » spécifiques dans une "direction» bien particulière, constituant ainsi un "aiguilleur» dont les conséquences se sont fait sentir jusque dans l'orientation complexe de l'évolution de la société occidentale. Isoler ces facteurs d'influence est l'une des tâches les plus difficiles de la sociologie de la culture : intérêts et idées se mêlent à de multiples titres. Pour Weber, la légitimation morale de l'autonomisation de la «sphère » de comportement économique est primordiale. La rupture avec la tradition provoquée par le protestantisme implique, d'une part, le rejet radical de toute forme de magie comme instrument de contrôle des puissances transcendantes et, d'autre part, l'abolition de tous les pouvoirs hiérocratiques. De là découle l'adoption de critères de rationalité spécifiquement fonctionnels comme moyens d'orientation de l'action et l'abandon des instances globales d'intégration des conceptions de valeur et de codification de ces dernières dans des normes d'action susceptibles d'être sanctionnées. Ces deux conséquences vont dans le sens d'une différenciation de "sphères » et d'une adhésion nouvelle à des critères de rationalité spécifique au sein de celles-ci. 
Dans l'éthique protestante, on rencontre une "séparation des sphères " particulière, dans la mesure où la valorisation positive de l'activité professionnelle est distincte des conséquences de cette activité. Le succès économique en tant que tel n'a pas à être justifié puisqu'il est d'emblée justifié par l'excellence éthique du travail professionnel. Tandis qu'auparavant, la richesse devait être légitimée, elle devient désormais neutre d'un point de vue éthique, elle n'est rien de plus que le résultat de l'éthique professionnelle. Cette dissociation entre l'appréciation morale des conséquences de l'action et l'appréciation morale de l'action en tant que telle pourrait être l'un des "aiguilleurs» qui ont contribué à l'importance culturelle du protestantisme en général. L'éthique protestante a réduit la pression interne de justification, elle a diminué la responsabilité vis-à-vis des conséquences de l'action économique. Que cette différenciation éthique soit ou non traduite sur le plan institutionnel, c'est là un autre problème. Que l'on aboutisse ainsi à une situation dans laquelle le lien social entre l'employeur et l'employé se réduit à un pur dédommagement du travail fourni, dans le cadre d'un contrat de travail formellement libre qui ne tient pas compte des conditions sociales de vie de l'employé, ou que l'on en arrive à une ouverture extrême de la liberté de disposition de l'employeur dans la combinaison des facteurs de production au sein de l'organisation de l'entreprise, ou, enfin, que l'employeur en vienne à se décharger des coûts des infrastructures et fasse peser sur la collectivité le coût des répercussions de la production industrielle: ce sont là, à chaque fois, des conséquences qui ne peuvent plus être simplement imputées à l'idée de l'éthique protestante du métier. Les intérêts idéels à la connaissance de leur état de grâce par les membres des sectes protestantes font place aux intérêts matériels à l'enrichissement personnel chez les «capitalistes» qui n'ont plus d'attaches religieuses. Une morale de virtuoses se transforme en morale quotidienne, l'innovation devient routine, le style individuel de conduite de vie devient un trait systématique.

Dans le cas du protestantisme, la différenciation des "sphères " a pu également être favorisée par la lutte que celui-ci a menée contre tous les pouvoirs hiérocratiques. Dans l'intérêt d'une intégration culturelle globale et d'une cure des âmes globale, proposée à tous les groupes de population, les instances ainsi abolies étaient auparavant tenues de servir d'intermédiaires dans des conflits entre les différents domaines de la vie, d'un point de vue idéel et d'un point de vue pratique. Les sectes, en revanche, peuvent être les porteurs d'idées beaucoup plus radicales et mettre entre parenthèses ou tolérer les conflits entre différents domaines de la vie. Dans tous les cas, le protestantisme ne dispose pas d'une instance d'intégration autonome; y compris dans le protestantisme non réformé, tout pasteur est son propre prophète et pape - s'il le veut et le peut. Par suite, du point de vue de la question sociale soulevée au XIX ${ }^{\mathrm{e}}$ siècle, le protestantisme n'a pas élaboré d'éthique sociale nouvelle qui aurait pu compenser les effets du capitalisme. Seul le mouvement ouvrier a développé une nouvelle morale sociale de solidarité collective, tandis que la bourgeoisie défendait l'idée du nationalisme collectif, non contraignante d'un point de vue social, les intérêts de la cohésion étatique et pour finir, l'idée d'une politique sociale et d'un Etat-Providence instrumentalisé, sans contenu social. Cependant, ces courants se sont fondés sur la " séparation des sphères " qui s'était auparavant imposée et sur l'imposition de critères de rationalité propres dont la validité s'affirme aujourd'hui encore de façon directe. La rentabilité économique et l'augmentation de la productivité sont devenues des objectifs systématiques. 
17 Max Weber souligne que sa caractérisation de l'éthique protestante revient seulement à mettre en avant "une composante particulière » qui ne se confond pas avec la " mentalité économique » dans son ensemble et n'est même pas susceptible d'expliquer la genèse $d u$ capitalisme comme système économique ${ }^{21}$. Les idées culturelles $d u$ protestantisme ascétique devraient être mises en relation avec "le rationalisme humaniste, avec les idéaux de vie et les influences culturelles de ce dernier, mais aussi [...] avec le développement de l'empirisme philosophique et scientifique, avec l'évolution de la technique et avec les biens culturels spirituels ${ }^{22}$ ». "Compte tenu de l'incroyable enchevêtrement d'influences réciproques entre les fondements matériels, les formes d'organisation sociales et politiques et le contenu spirituel des époques culturelles réformatrices ", la stratégie d'analyse ne saurait emprunter d'autre voie que celle-ci : isoler dans un premier temps un élément culturel et «tenter de mesurer la part qui revient à ces motifs religieux et celle qui revient à d'autres facteurs dans la genèse historique des contenus culturels modernes $»^{23}$. Ce programme et son application n'ont cessé de susciter de vives controverses. Mais elles ne remettent pas en cause l'importance de cette problématique, et ne peuvent résoudre les problèmes qu'elle soulève.

Les intérêts et les idées ne s'opposent pas frontalement. Les idées sont associées à des intérêts, elles doivent produire un "résultat». Les religions doivent être capables d'interpréter l'expérience spécifiquement «irrationnelle » qui est vécue par leurs adeptes; les normes juridiques servent, sous des formes diverses, à imposer des intérêts matériels. Inversement, les intérêts sont associés à des idées, ils sont orientés vers des objectifs et ont recours à des moyens dotés d'une légitimité. L'intérêt idéel d'un groupe à l'interprétation, à l'articulation et à la réalisation d'une idée devient en même temps un intérêt matériel lorsque le groupe cherche à l'utiliser pour gagner de l'influence et s'enrichir. Dans la lutte des compétences, l'interprétation et la "gestion » des idées peuvent aussi être monopolisées. Des délimitations de compétences se font jour et, par là, une différenciation du domaine de validité socialement contrôlé qui revient aux idées. Les intérêts des intellectuels à trouver la plus grande cohérence possible et, d'un point de vue cognitif, une absence de contradiction dans les idées qu'ils ont à interpréter, entrent en concurrence avec l'intérêt à l'applicabilité pratique des idées chez ceux qui sont censés les réaliser dans la vie. Les intérêts et les idées renvoient ainsi à deux perspectives d'analyse. "L'une et l'autre sont également possibles $^{24} »$ : c'est sur ces mots que se conclut l'étude de Max Weber sur l'éthique protestante. Cependant, ces deux perspectives doivent être maintenues dans leur complémentarité. Ce n'est qu'à cette condition que les recherches comparatives sur les sociétés et les cultures peuvent être à même de saisir les différences constitutives entre les sociétés. "Des formes d'organisation économique qui sont extérieurement identiques peuvent être conjuguées à une éthique économique très différente et elles produisent alors des effets historiques très différents selon les particularités de cette éthique ${ }^{25}$. " Aujourd'hui encore, il peut exister des différences entre des sociétés qui partagent un même degré de développement technique et économique, comme l'a montré l'échec relatif de la thèse de la convergence.

Les idées constituent des sphères de valeurs spécifiques et déterminent les orientations de l'action à l'intérieur de ces sphères. Telle était l'hypothèse de Weber. Par exemple, à travers une éthique spécifique du travail, elles peuvent établir une séparation entre la sphère du travail et celle de la consommation, entre l'entreprise et la famille, dissocier 
rapports de travail et rapports privés et conférer à la sphère du travail un contenu de valeur autonome. Par l'institutionnalisation, la sphère de validité est elle aussi isolée socialement. Ce facteur exerce une influence sur la perception et impose des limites à l'action. La dépense et le profit ne sont plus calculés que dans le cadre d'un contexte d'action institutionnalisé. Les autres coûts et profits sont externalisés et ne sont pas imputés au même contexte d'action. Selon les limites fixées à ce contexte d'action, les «bilans» obtenus varient considérablement. Une usine de radios déficitaire fait faillite; au sein d'un conglomérat, la même unité de production peut être valorisée positivement si elle complète une palette d'offres. Les Etats-nations peuvent maximiser leurs profits en fonction de critères internes tout en faisant obstacle à leur développement économique global au sein des réseaux de l'économie mondiale. L'unité que vise l'apport de légitimité politique, l'Etat-nation, peut être dotée d'une légitimité institutionnelle mais représenter un «mauvais » cadre de référence d'un point de vue fonctionnel. Seuls de nouveaux fondements de valeur peuvent opérer une médiation entre des contextes d'action institutionnalisés et les sphères de valeur, ou, le cas échéant, les bouleverser. Les changements dans les rôles sexués, la construction d'une Europe occidentale unifiée, la mise en œuvre du désarmement ou le contrôle de l'économie atomique ne découlent manifestement pas de façon automatique du croisement des intérêts. Sans idées pour fonder les valeurs, les efforts pour trouver un compromis entre les intérêts demeurent vains. En ce sens aussi, les idées qui font défaut jouent le rôle d'« aiguilleurs" ".

\section{BIBLIOGRAPHIE}

Les abréviations utilisées dans le texte pour désigner les œuvres de Max Weber renvoient aux textes suivants :

GARS I : Gesammelte Aufsätze zur Religionssoziologie I, $7^{\mathrm{e}}$ éd., Tübingen, 1978.

[Traduction française utilisée : L'Ethique protestante et l'esprit du capitalisme, trad. I. Kalinowski, Paris : Flammarion, 2000.

Pour l' « Introduction », Sociologie des religions, trad. J. P. Grossein, Paris : Gallimard, 1996.]

PE II : Die protestantische Ethik II. Kritiken und Antikritiken, éd. par J. Winckelmann, $4^{\mathrm{e}}$ éd., Gütersloh, 1982.

[Traduction française in : L'Ethique protestante et l'esprit du capitalisme, trad. J. P. Grossein, Paris : Gallimard, 2004.]

Sur le déploiement de la mentalité capitaliste, voir aussi

Max Weber : Wirtschaftsgeschichte, $3^{\mathrm{e}}$ éd., Berlin, 1958, p. 300-315.

[Trad. fr. : Histoire économique, trad. C. Bouchindhomme, Paris : Gallimard, 1991, p. 295 sq.]

et, en complément, le chapitre de sociologie de la religion dans

Max Weber : Wirtschaft und Gesellschaft [Economie et société], 5éd., Tübingen, 1972, p. 245-381.

[Trad. fr. : Sociologie de la religion, trad. I. Kalinowski, Paris : Flammarion, 2006.]

La bibliographie relative à L'Ethique protestante est abondante. Nous ne mentionnerons ici que quelques titres importants en nous concentrant sur les thèmes abordés dans cet article. 
La référence en matière de bibliographie, jusqu'à la date de 1976, est le recueil de Constans Seyfarth et Gert Schmidt : Max Weber Bibliographie : Eine Dokumentation der Sekundärliteratur, Stuttgart, 1977.

En premier lieu, citons Reinhard Bendix : Max Weber - das Werk, Munich, 1964, qui a été le premier à replacer les études sur le protestantisme dans le système de la sociologie wébérienne.

Constans Seyfarth et Walter M. Sprondel ont édité sous le titre Seminar : Religion und gesellschaftliche Entwicklung, Studien zur Protestantismus-Studie Max Webers [Religion et évolution sociale. L'étude de Max Weber sur le protestantisme], Francfort, 1973, une série de contributions anciennes et récentes relatives à L'Ethique ; parmi elles, celles des deux directeurs du volume présentent un intérêt particulier du point de vue des questions qui nous occupent ici.

Johannes Weiß : Max Webers Grundlegung der Soziologie [Le fondement de la sociologie selon Max Weber], Munich, 1975, replace L'Ethique dans le cadre de la thèse sur la rationalisation.

Les essais de reconstitution des thèses de Weber les plus récents sont ceux de Gordon Marshall : In Search of the Spirit of Capitalism : An Essay on Max Weber's Protestant Ethic Thesis, Londres, 1982, et Gianfranco Poggi, Calvinism and the Capitalist Spirit, Amherst, 1983.

Les travaux de Wolfgang Schluchter sont fondamentaux pour étudier le développement des analyses wébériennes : Rationalismus der Weltbeherrschung [Le rationalisme de la maîtrise du monde], Francfort, 1980 (notamment les deux premiers articles, « Die Paradoxie der Rationalisierung » [Le paradoxe de la rationalisation] et "Wertfreiheit und Verantwortungsethik » [Non-imposition des valeurs et éthique de la responsabilité] ; Die Entwicklung des okzidentalen Rationalismus [Le développement du rationalisme occidental], Tübingen, 1979 (notamment le chapitre 6) ; mentionnons encore ses introductions systématiques aux collectifs qu'il a édités : Max Webers Studie über das antike Judentum [L'Etude de Max Weber sur le Judaïsme antique], Francfort, 1981 ; Max Webers Studie über Konfuzianismus und Taoismus [L'Etude de Max Weber sur Confucianisme et taoïsme], Francfort, 1983 ; Max Webers Studie über Hinduismus und Buddhismus [L'Etude de Max Weber sur Hindouisme et bouddhisme], Francfort, 1984 ; Max Webers Sicht des antiken Christentums [La vision du christianisme ancien chez Max Weber], Francfort, 1985.

Note de la rédaction : En raison de l'abondance de publications sur Max Weber, les indications bibliographiques données par l'auteur en 1986 n'ont pas été actualisées et complétées.

\section{NOTES}

1. GARS I, p. 252 [trad. fr. $1996:$ p. 349 sq].

2. GARS I, p. 1 [trad. fr. 2000, p. 49].

3. GARS I, p. 12 [trad. fr. 2000, p. 63].

4. GARS I, p. 12 [trad. fr. 2000, p. 63].

5. Ibid.

6. GARS I, p. 82 [trad. fr. 2000, p. 150].

7. GARS I, p. 29 sq. [trad. fr. 2000, p. 85].

8. PE II, p. 307 [trad.fr. 2003, p. 422 (traduction légèrement modifiée)].

9. PE II, p. 307 sq. [trad. fr. 2003, p. 381 sq.].

10. Cf. PE II, p. 314 sq. [trad. fr. 2003, p. 431 sq.].

11. GARS I, p. 20 [trad. fr. 2000, p. 73].

12. Cf. GARS I, p. 190-193 [trad. fr. 2000, p. 284-288].

13. Cf. PE II, p. 310 sq. et GARS I, p. 210 sq. [trad. fr. 2000 , p. 308 sq.].

14. PE II, p. 313 [trad. fr. 2003, p. 430].

15. PE II, p. 318 [trad. fr. 2003, p. 436 (traduction légèrement modifiée)].

16. PE II, p. 319 [trad. fr. 2003, p. 437 (traduction légèrement modifiée)]. 
17. PE II, p. 168 [trad. fr. 2003, p. 370].

18. Cf. PE II, p. 319 [trad. fr. 2003, p. 437].

19. GARS I, p. 37 sq. [trad. fr. 2000, p. 93].

20. PE II, p. 319 [trad. fr. 2003, p. 437].

21. Cf. PE II, p. 169 [trad. fr. 2003, p. 371 sq.].

22. GARS I, p. 205 [trad. fr. 2000, p. 302].

23. GARS I, p. 83 [trad. fr. 2000, p. 152]

24. GARS I, p. 205 [trad. fr. 2000, p. 303].

25. GARS I, p.238 [trad. fr. 1996, p. 332 (traduction légèrement modifiée)].

INDEX

Schlüsselwörter : Ideen, Interessen, Kultursoziologie, Max Weber, protestantische Ethik

Mots-clés : éthique protestante, idées, intérêts, Max Weber, sociologie de la culture

\section{AUTEURS}

\section{RAINER LEPSIUS}

M. Rainer Lepsius (né en 1928) est professeur émérite de sociologie à l'Université de Heidelberg. Pour plus d'informations, voir la notice suivante. 